\title{
Heat Transfer Characteristics during Quench of High Temperature Solid*
}

\author{
Masanori MONDE** \\ ${ }^{* *}$ Institute of Ocean Energy, Saga University, \\ 1 Honjo-machi, Saga 840-8502, Japan \\ E-mail: monde@me.saga-u.ac.jp
}

\begin{abstract}
This paper reviews characteristics of heat transfer during quenching high temperature body using liquid jet impingement. The temperature of body is initially kept higher than the Leidenfrost temperature. The flow pattern dramatically changes with a decrease in the surface temperature. At a moment of jet impingement, the liquid is randomly splashed away. After that, it seems to change a cone shape splashed flow, in which liquid is confirmed to be contact with the surface in an impinging zone. Finally, the liquid contact area where occurs rigorous boiling starts moving radially. Under such flow configuration, how surface temperature and heat flux change with time and homogenous nucleation for vapor generation are discussed.
\end{abstract}

Key words: Quench, High Temperature, Heat Transfer, Jet Impingement, Homogeneous Nucleation

\section{Introduction}

Quenching is widely used and has been extensively studied for controlling the mechanical and metallurgical properties of materials in the manufacturing industry. Recently, it is important for cooling of high power electronic chips. Also, during a loss of coolant accident (LOCA) in a nuclear power plant, quenching can be used as an effective cooling process. In addition, high density cooling technology is focused in designing plasma facing surface in fusion reactor. In spite of these important applications, understanding of the field of quenching and wetting front propagation is far from a mature science from the following reason that during quenching high temperature material, different cooling modes such as film boiling, nucleate boiling and transient from film to nucleate boilings coexist on the surface and these modes changes with time. Especially, during the transient the surface temperature and surface heat flux dramatically change, which are hardly measured and then how these changes have respect to wetting of the surface and flow configuration on the surface is not clear.

Recently, Monde et al. [1-3] had developed inverse solution to estimate surface temperature and heat flux from measured temperatures in the body during quench of high temperature one and made it possible what happens on the surface during these changes, correctly.

In this review paper, author focuses on the quench cooling with an impinging water jet and summarizes results, which have been published by Monde and his coworkers[4-10] until now and finally discusses relationship between beginning of wetting and homogenous nucleation and a repeated cycle of wetting, film boiling and rewetting which has been speculated to occur during this time. 


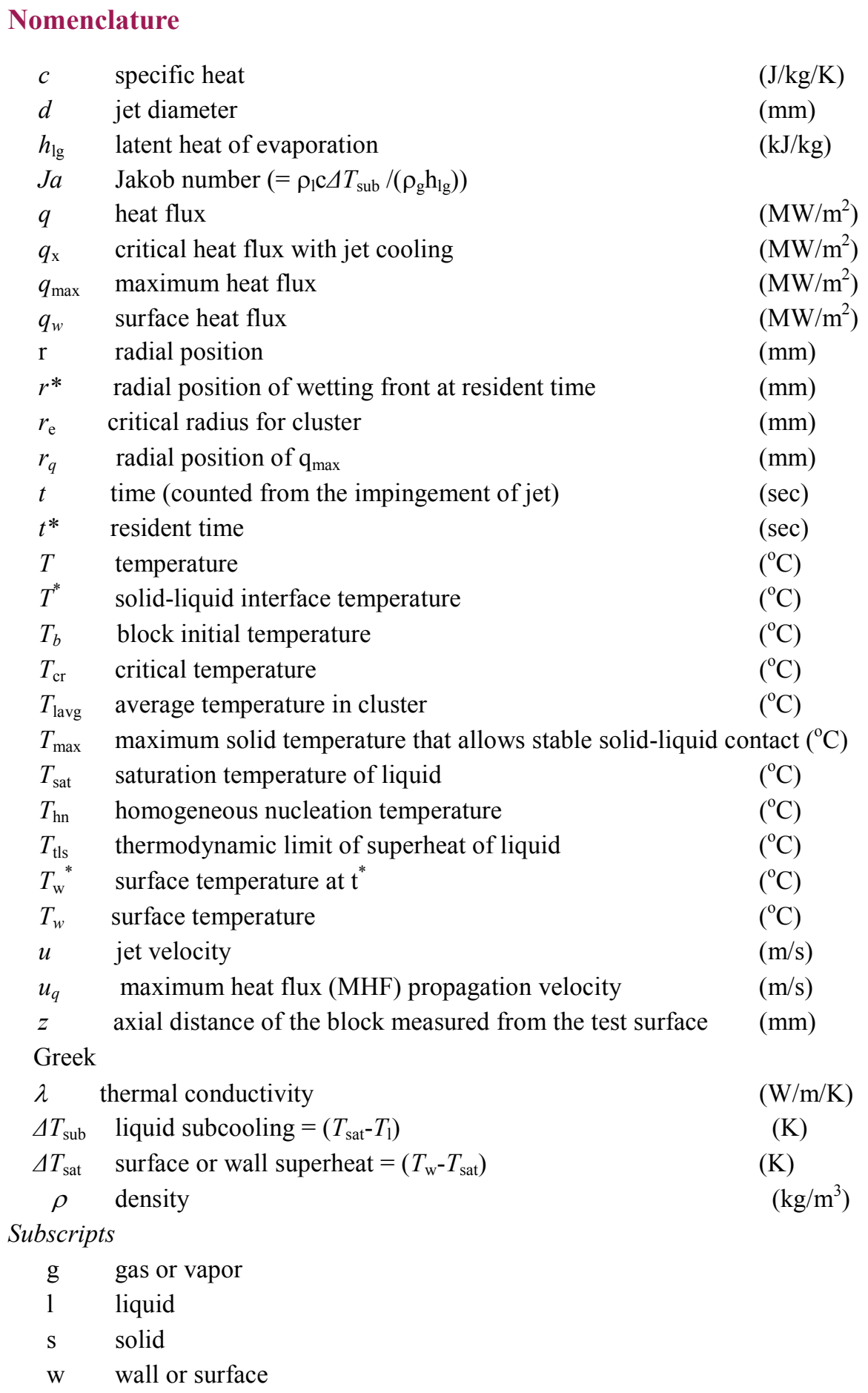

\section{Quench of high temperature surface with impinging jet}

\subsection{Flow situation on high temperature surface during quenching}

Figures 1 shows a sequence of video clips at the early stages (from $3 \mathrm{~ms}$ to $3 \mathrm{~s}$ ) during quenching of a steel block and brass block by a water jet. Figure $1(\mathrm{a})$ is for the steel $\left(T_{\mathrm{b}}=\right.$ $\left.500{ }^{\circ} \mathrm{C}\right)$ and Fig. $1(\mathrm{~b})$ for the brass $\left(550{ }^{\circ} \mathrm{C}\right)$ by a $5 \mathrm{~m} / \mathrm{s}-50{ }^{\circ} \mathrm{C}$ jet.

Soon after jet impingement, a circular shiny (mirror like) liquid sheet was observed from Fig. 1(a) at the center of the block as shown up to $30 \mathrm{~ms}$ (for some other experimental conditions this phenomenon persisted for several seconds). During this period, the flow was 
very calm and quiet and no boiling sound was heard by an unaided ear. It seems that the jet hardly made contact with the surface at this stage. At the start there may be a brief solid-liquid contact at the center where bubbles form, coalesce and make the jet slide over the hot surface resulting in a flat sheet. When the size of the shiny sheet reaches its limit, numerous tiny liquid droplets form at its fringe as shown in the figure for $30 \mathrm{~ms}$. A little later (around $200 \mathrm{~ms}$ ), the above mentioned shiny liquid sheet started disappearing and the jet contacted the surface directly. Boiling might have occurred and some liquid was found to be splashed away at an angle from the surface as shown in Fig. 1(a) at $200 \mathrm{~ms}$ and 500 $\mathrm{ms}$. More rigorous contact was evident after $500 \mathrm{~ms}$ and a growing wet patch formed at the center. The liquid was deflected more as time progressed because of the possible interaction between the jet and the bubbles generated in the wetted area. At 2 and $4 \mathrm{~s}$, the wet front, where rigorous splashed droplet was observed, is moving toward the outside.

On the other hand, Fig. 1(b) exhibits a completely different sequence of video images during $3 \mathrm{~ms}$ to $4 \mathrm{~s}$ of quenching of a brass block initially heated to $550{ }^{\circ} \mathrm{C}$. Within $30 \mathrm{~ms}$, the flow was found explosive and noisy where splashed liquid moved away in all possible directions. During 200 - $300 \mathrm{~ms}$, oscillating sheet flow was observed. After about $500 \mathrm{~ms}$ a nearly stable sheet flow was evident. Unlike the flow for steel as shown in Fig. 1(a), no wet patch was found in the early stages of quenching. It took about $170 \mathrm{~s}$ to form a growing wet patch and to have a flow pattern the same as shown in Fig. 1(a) for $500 \mathrm{~ms}$. It should be mentioned finally that the time duration until the wet patch starts growing strongly depends on the quenched material and thermal-hydraulic condition of the jet.

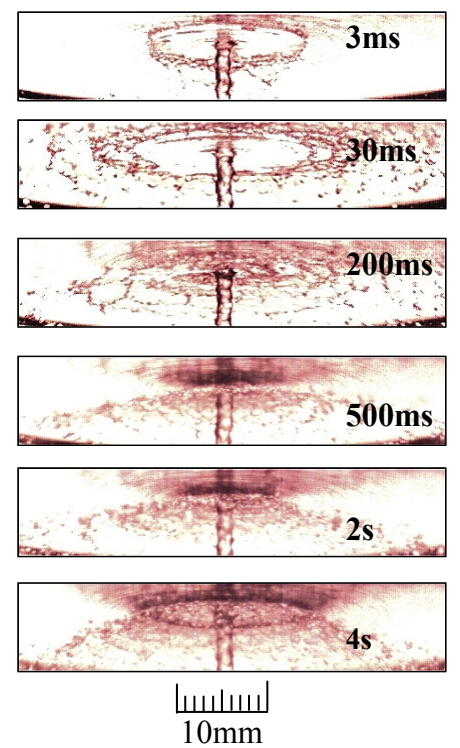

( a ) Steel
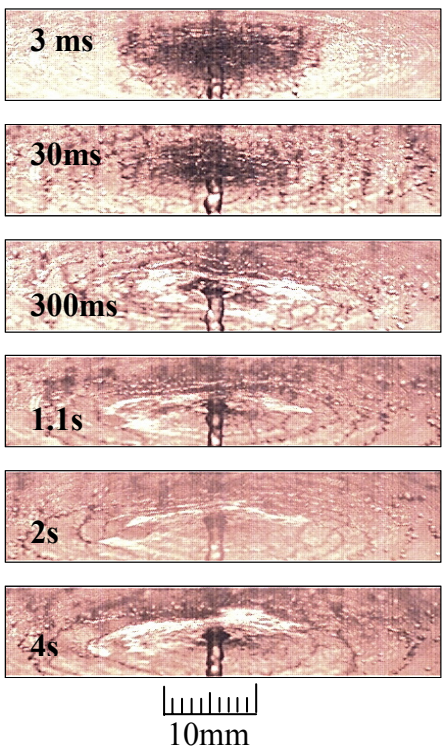

( b ) Brass

Fig.1 Video clips during quenching of steel and brass blocks (Ref.[10])

\subsection{Surface temperature and heat flux changes during quenching}

Figure 2 shows an example of the surface temperature and heat flux distributions with time and radial position estimated from the inverse solution with the change of flow pattern such as shown in Fig. 1.

The cooling curve in Fig. 2 shows that the temperature close to the center of the test piece drops from 400 to $100^{\circ} \mathrm{C}$ within 2 seconds after the jet first strikes. During this time, the heat flux increases drastically and reaches its maximum value, while the surface temperature sharply decreases. Therefore, process of heat transfer until the maximum heat flux is reached corresponds to the transition from film boiling to nucleate boiling. In other words, the surface begins being wetted partially and finally is stably wetted to ensure the 
maximum heat flux. Beyond the maximum heat flux, the surface is surely wetted enough to generate nucleate boiling. The maximum heat flux location moves outwards in the radial direction and then the wetted surface area also enlarged. For this case, it takes about 10 seconds for the maximum heat flux position to reach the outer end surface. The quenching process completely finishes at about 10 seconds, and the surface temperature is fully cooled down lower than $100^{\circ} \mathrm{C}$.
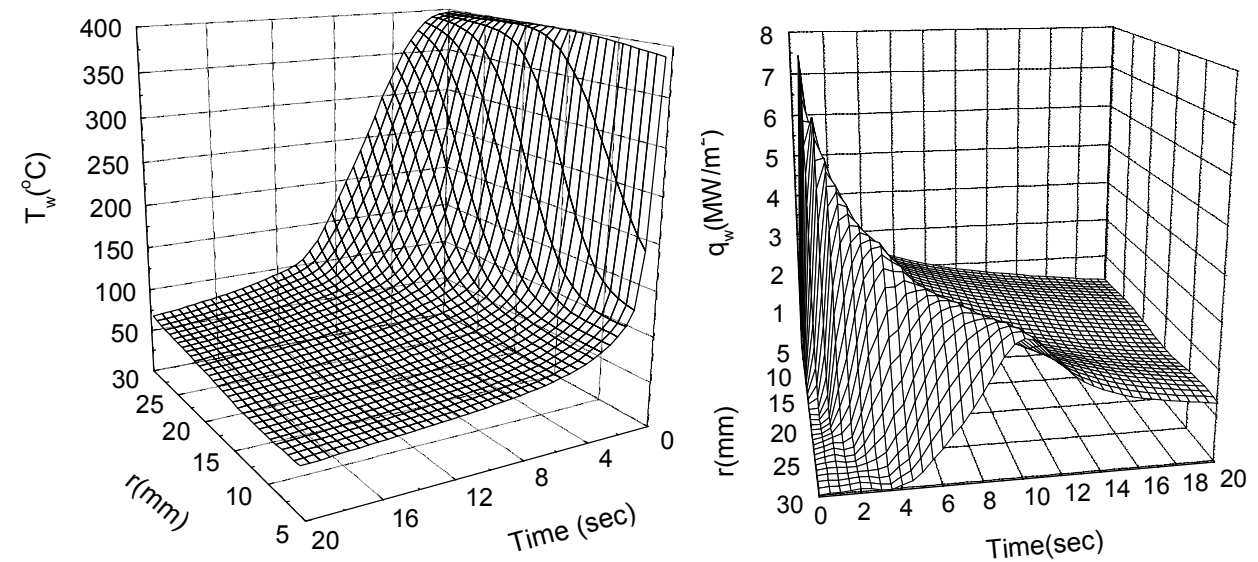

Fig. 2. Cooling curve and heat flux distribution during quenching for brass

$$
\left(T_{\mathrm{b}}=400^{\circ} \mathrm{C}, \Delta T_{\mathrm{sub}}=80 \mathrm{~K}, u=15 \mathrm{~m} / \mathrm{s}\right)(\operatorname{Ref} .[8])
$$

Figure 3 shows the change in the surface temperature and heat flux in the flow configuration at a time of $t=4.3 \mathrm{sec}$ after the jet impingement. The two dot lines for the temperatures in Fig. 3 shows the measured temperatures at the positions of 2.1 and $5.0 \mathrm{~mm}$ in depth, from which the surface temperature and heat flux are estimated using the Monde inverse solution method.

The flow situation can be divided into three regions; (a) no visible boiling $(r=0-12$ $\mathrm{mm}$ ) (b) vigorous boiling heat transfer $(r=12-18 \mathrm{~mm})$ and (c) dry region $(r=18-45$ $\mathrm{mm}$ ). In the region with no visible boiling activity, boiling is suppressed by forced convection or the surface temperature is cooled down not to generate boiling. This region corresponds to the end of the quenching, which appears at a slightly upstream of the vigorous boiling region in the zone marked (2).

The maximum heat flux point appears at a radial position of $13.6 \mathrm{~mm}(\approx 14 \mathrm{~mm})$, which is just inside the vigorous boiling region as shown in Fig. 3. As mentioned above, the region with $r=10$ to $12 \mathrm{~mm}$ belongs to the no visible boiling region but since the surface temperature is very high, it is expected that nucleate boiling occurs but bubbles collapse quickly due to the high sub-cooling and consequently no vigorous boiling is observed. Thus in terms of the different modes of heat transfer the image in Fig. 3 may be interpreted as (i) single phase convection $(0-10 \mathrm{~mm})$, (ii) nucleation boiling $(10-14$ $\mathrm{mm}$ ), (iii) transition boiling $(14-18 \mathrm{~mm}$ ) and (iv) single phase convection to vapor phase plus radiation $(18-47 \mathrm{~mm})$.

In the single phase convection region the surface temperature is close to the saturation point. For example at the position marked (3) in Fig. 3 the superheat is about $5 \mathrm{~K}$, which is reasonable for single phase forced convection heat transfer. At the point (2) the surface superheat has increased to $18 \mathrm{~K}$ and heat flux increases monotonically with radial position towards the maximum, which is consistent with nucleation boiling in subcooled forced convection. At the maximum heat flux point, the surface superheat is $41 \mathrm{~K}$. This again is higher than typical values for saturated nucleate pool boiling but reasonable for jet 
impingement with a subcooled liquid. In the dry region the surface temperature climbs rapidly from about $250^{\circ} \mathrm{C}$ to the block initial temperature of $400^{\circ} \mathrm{C}$ and the heat flux becomes very small.
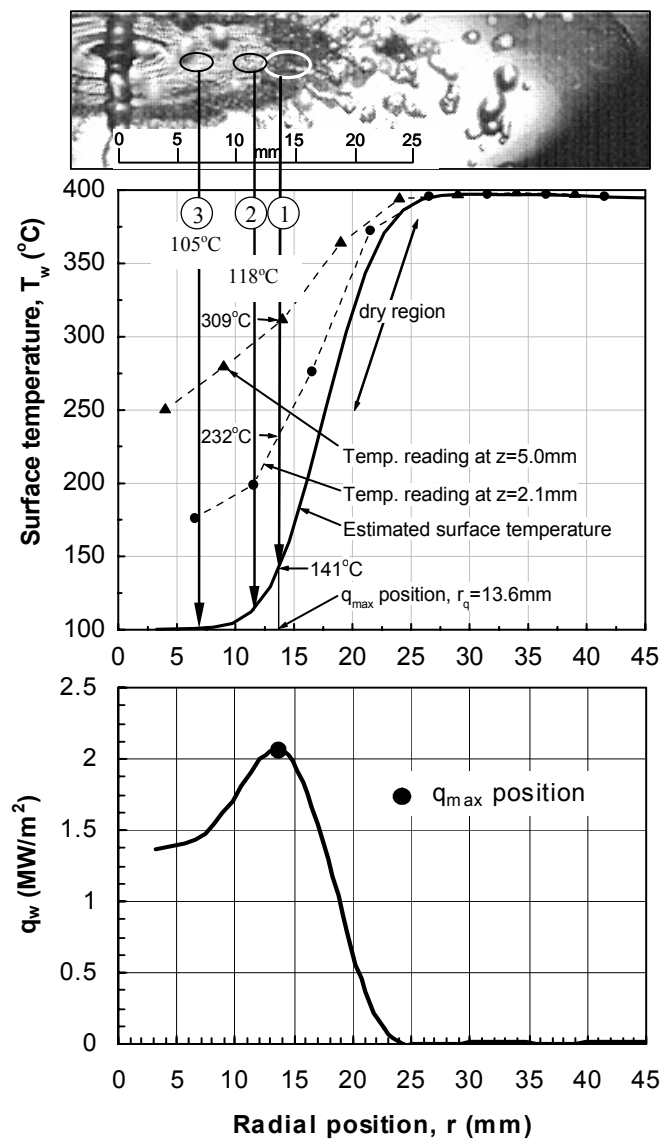

Fig. 3: Regimes of boiling and maximum heat flux during wetting front propagation (Steel: $T_{\mathrm{b}}=400^{\circ} \mathrm{C}, \Delta T_{\text {sub }}=50 \mathrm{~K}, u=3 \mathrm{~m} / \mathrm{s}, t=4.3 \mathrm{sec}$ ) (Ref.[8])

When one focuses a mode of boiling at a point on the surface during moving of the wetting front, it will experience different boiling modes at different times depending on its position relative to the wetting front such that the mode changes from film boiling, transition boiling, nucleate boiling and finally single phase. The maximum heat flux at each point serves as the boundary between transition boiling and nucleate boiling

It may be worth mentioning that the surface temperature varies around $150-180{ }^{\circ} \mathrm{C}$ (for $r=8-30 \mathrm{~mm}$ in the case of $T_{\mathrm{b}}=400{ }^{\circ} \mathrm{C}, \Delta T_{\text {sub }}=80 \mathrm{~K}, u=15 \mathrm{~m} / \mathrm{s}$ ) at the occurrence of the maximum heat flux. In other words, the maximum heat flux appears when a heat supply from the solid just balances cooling limit of liquid, which is decreasing with the radial position.

Filiporic et al.[11] carried out quench of high temperature surface using a wall jet and pointed out that a repetition of wet and dry, which corresponds to transition boiling, takes place and then the maximum heat flux is reached.

\subsection{Characteristics of flow pattern and audible sound on cooling curve}

Figure 4 shows a flow pattern identified from video image. It may be worth mentioning that the sequence of the flow pattern is totally identical except that the time when the same flow pattern appears during quench is strongly influenced by the initial temperature, liquid 
subcooling, jet velocity and thermal properties of test material.

Figure 4 depicts the flow pattern to identify six different types from photos A to F, which can be categorized as follows:

Type A: Highly chaotic two-phase flow occurs as soon as the water jet is impinged on a hot surface. Tiny bubbles may be formed during brief instants of solid-liquid contact. Intensity and existence of chaotic depends on the higher value of the thermal parameter, $\rho c \lambda$, of the material and the higher surface temperature.

Type B: A stable film boiling is sustained without a brief solid-liquid contact at the center where bubbles form, coalesce and make the jet slide over it resulting in a flat liquid sheet. At a radius of about $10 \mathrm{~mm}$, the sheet breaks into droplets that move outwards at angles of 5 -10 degrees from the surface.

Type C: As time passes on, the surface temperature goes down due to the heat transfer. A small longer solid-liquid contact is unstably established, by which a considerable number of isolated bubbles are irregularly formed that compels the jet to be directed outward with splashed liquid droplets.

Type D: This is a conical sheet flow pattern observed, which is a delicate flow pattern occurring under favorable thermal-hydraulic conditions. It seems that a small wet patch (larger than that for the type C) remains in contact with the surface for a considerable period of time at the center and helps produce more bubbles that deflect the jet further from the impinging surface and make an angle of about 15 - 20 degrees.

Type E: The stable wet patch grows more having a size larger than about double of the jet diameter and stagnates for a certain time, helps generate more and more bubbles near its fringe.
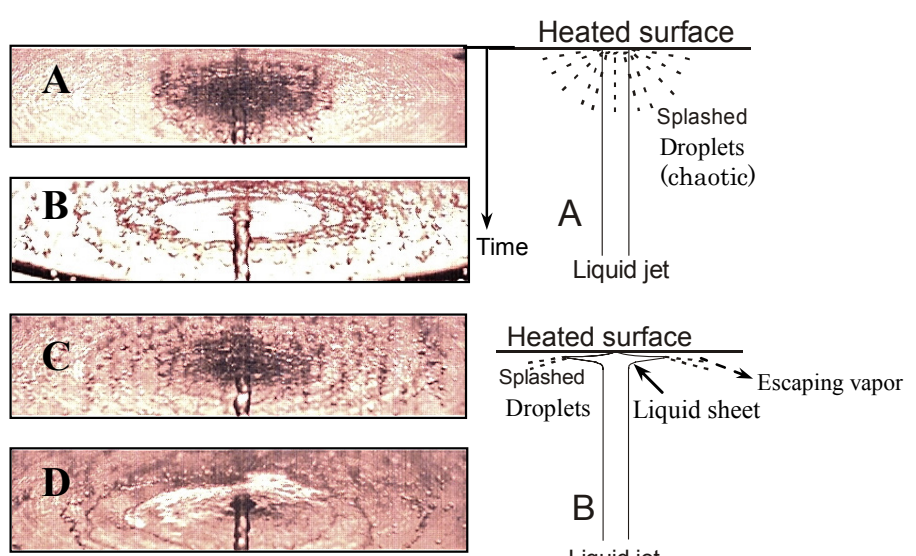

Droplets Liquid sheet
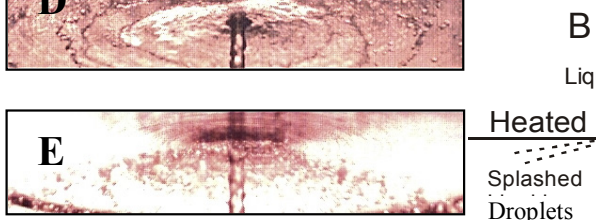

Liquid jet

Heated surface
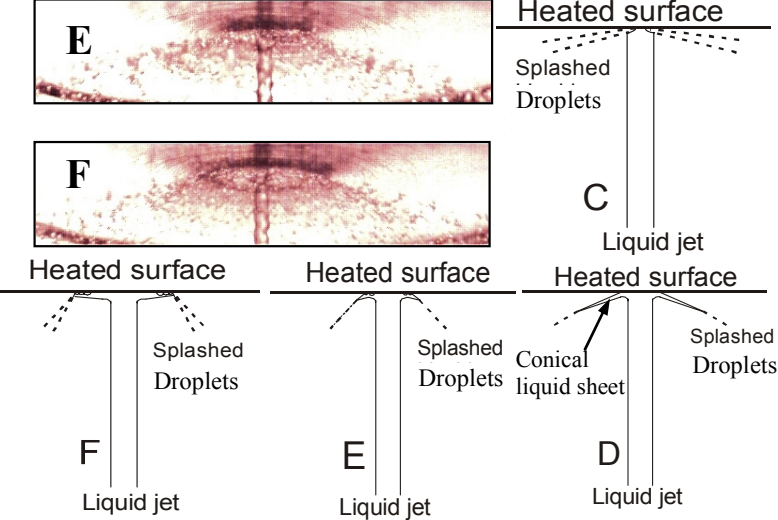

Heated surface

$$
\text { Liquid jet }
$$
Heated surface

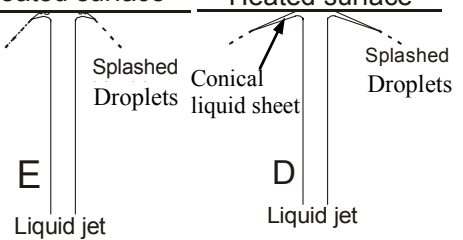

Fig.4 Flow patterns identified from video observation and flow model (Ref. [10]) 
Type F: As the surface temperature of the wet patch goes down further, the heat supply from the solid surface reaches smaller than the liquid cooling. Finally, the wet patch starts growing along the radial direction. The boiling modes between types $\mathrm{E}$ and $\mathrm{F}$ are identical except for a magnitude of the heat flux.

A detail explanation is given in Ref.[10].

Figure 5 shows a measured audible sound for a different quench shown in Figure 4. As mentioned before that the sequence of the flow pattern is totally identical except that the time, it may be interesting to compare the change in between flow pattern and audible sound during the quench. The type A appears too short to identify the audible sound. During time that the sheet structure is present the boiling sound becomes very quiet until first 50 $\mathrm{sec}$ and from 120 to $220 \mathrm{sec}$ in Fig.5 (Patterns B and D in Fig.4). Between patterns B and D, the liquid is violently splashed away by a strong vapor generation, by which a noisy sound may be generated. This sound from 50 to $120 \mathrm{sec}$ could be heard as a sharp splattering sound. After the pattern D, the sheet disappeared and then violent splashed droplet flow is generated, making another noisy sound occur as shown by Type E, and the wetting front starts moving forward over the surface. During the movement of wetting front, the noisy sound is accompanied. The heat transfer mode is finally approaching to forced convection and its sound disappears.

The large amplitude of sound signal means that a repetition of wet and dry surfaces takes place at very short period. On the other hand, the measured temperature does not indicate any change, because a short period of temperature change does not penetrate to the thermocouple sensor location.

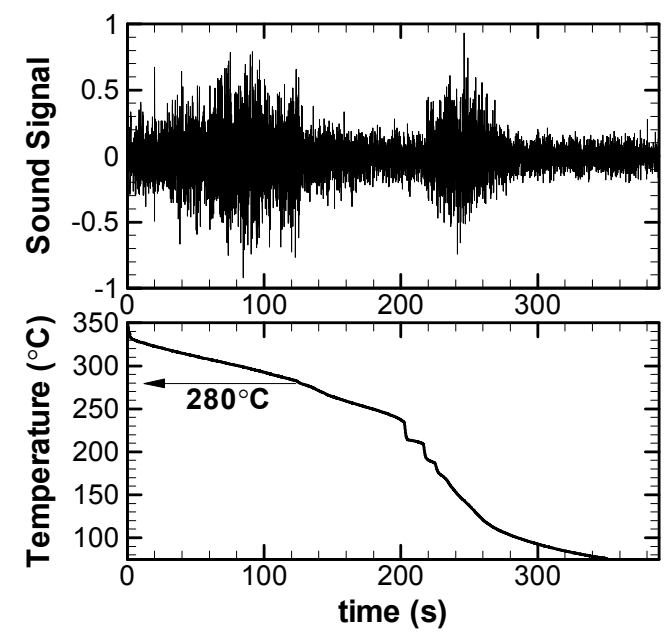

Fig. 5 Audible sound during quench and corresponding cooling curve (embedded thermocouple reading: $4 \mathrm{~mm}$ from center, $5 \mathrm{~mm}$ from surface, Copper, $T_{b}=350{ }^{\circ} \mathrm{C}, T_{l i q}=49$ $\left.{ }^{\circ} \mathrm{C}, u=3 \mathrm{~m} / \mathrm{s}\right)($ Ref.[7])

\subsection{Start time of wetting}

It is confirmed that as shown in Fig.5, the explosive sound is lasting after water jet impinges on the hot surface, and then the position of wetting front starts moving outward after some time during which the sound becomes lightly calm. Figure 6 shows the resident time, $t^{*}$, at which the wetting front starts moving outward plotted against $u \Delta T_{\text {sub }}$, which may play a role of intense of cooling.

It is found from Fig. 6 that as the value of $u \Delta T_{\text {sub }}$ decreases, the resident time becomes longer. In addition, for the case of $t^{*}>30 \mathrm{sec}$, there is a relationship between $t^{*}$ and $u \Delta T_{\text {sub. }}$. 
The resident time strongly depends on a capacity for cooling the surface and for supplying heat from solid. The mechanism for the resident time is not clearly understood, yet. Incidentally, Piggott et al.[12] pointed out the existence of the resident time and that its time is a strong function of water subcooling, jet velocity, heat generation rate, and thermal conductivity.

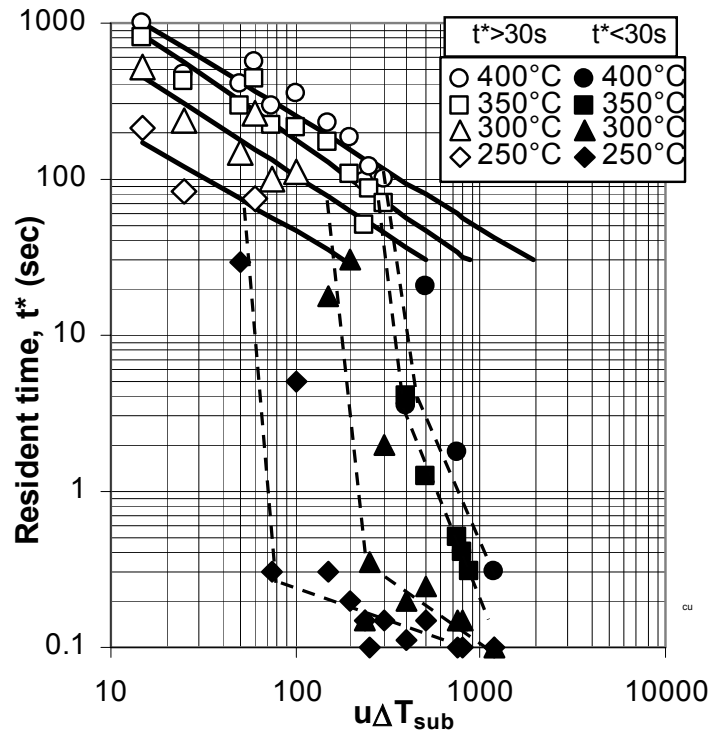

Fig.6 Resident time, $t^{*}$ at different initial temperatures for copper plotted against $u \Delta T_{\text {sub }}$ (Ref. [6])

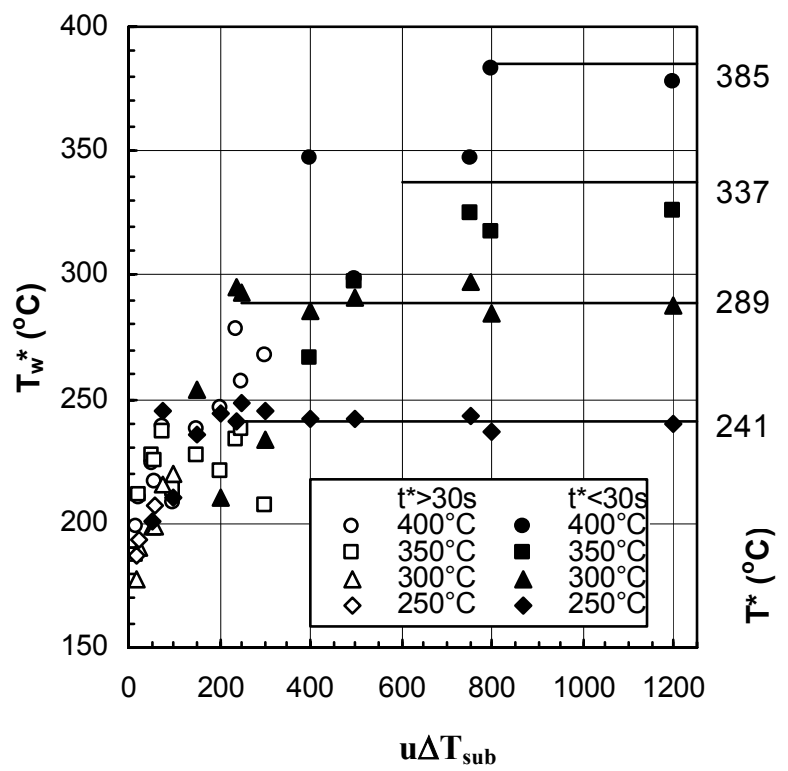

Fig.7 Surface temperature for copper at resident time $t^{*}$

\subsection{Surface temperature at resident time}

Figure 7 shows a relationship for copper between the surface temperature at the resident

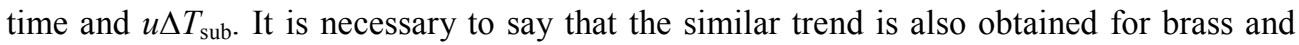
carbon steel. The straight line in Fig. 7 is the surface temperature at a moment when semi-infinite liquid at high temperature of $T_{\text {liq }}$ contacts semi-infinite body at high temperature of $T_{\mathrm{b}}$. and its temperature is given as follows: 


$$
T^{*}=\frac{T_{b}-T_{l i q}}{1+1 / \beta}+T_{l i q}, \quad \beta=\sqrt{(\rho c \lambda)_{s} /(\rho c \lambda)_{l}}
$$

For the case of $t^{*}>30 \mathrm{sec}$, even though a stable liquid-solid contact is confirmed within an impinging zone on the surface, the condition that the contact area does not extend is lasting until the resident time. The reason that the contact area does not extend is that the heat flux at the front of the contact area is enough high to splash all of water out. On the other hand, for the case of $t^{*}<30 \mathrm{sec}$, the wetted area extends so fast immediately after the impingement of water jet and the surface temperature at that time is almost constant for any value of $u \Delta T_{\text {sub. }}$ Quick expansion of the contact area usually occurs for the relatively lower initial temperature and smaller value of $(\rho c \lambda)_{s}$, since the heat supply from the solid is not enough. Its value is approaching to the temperature given by Eq.(1). The reason that the temperature of $T_{\mathrm{w}}{ }^{*}$ is close to the temperature of $T^{*}$ for the case of $t^{*}<30 \mathrm{sec}$, seems to be due to a small number of repetitions for liquid-solid contact during a short resident time according to the change in the sound intensity as shown in Fig.5.

\subsection{Correlation for surface temperature at wetting start time}

Figure 7 indicates that there is a regular relation between the time of wetting and the surface temperature. In order to tentatively determine the relationship, one applies the least mean square method to it, giving the following equations.

$$
\begin{gathered}
\frac{\sqrt{(\rho c \lambda)_{s} / t^{*}}\left(T_{w}^{*}-T_{\text {sat }}\right)}{(\rho c u)_{l}\left(T_{\text {sat }}-T_{\text {liq }}\right)}=14\left(\frac{2 r^{*}}{d}\right)^{0.72} \\
\left\{(\rho c u)_{l}\left(T_{\text {sat }}-T_{\text {liq }}\right)\right\}^{-0.41}\left(T_{b}-T_{\text {liq }}\right)^{-0.99} \\
\frac{T_{w} *-T_{\text {sat }}}{T_{b}-T_{\text {liq }}}=157\left(\frac{(\rho c \lambda)_{s}}{(\rho c \lambda)_{l}}\right)^{-0.49}\left(\frac{2 r^{*}}{d}\right)^{-0.49} \\
\left\{(\rho c u)_{l}\left(T_{\text {sat }}-T_{\text {liq }}\right)\right\}^{0.14}\left(T_{b}-T_{\text {liq }}\right)^{-0.68}
\end{gathered}
$$

Where $r^{*}$ is location of the wetting front at which the temperature of $T_{\mathrm{w}} *$ is obtained.

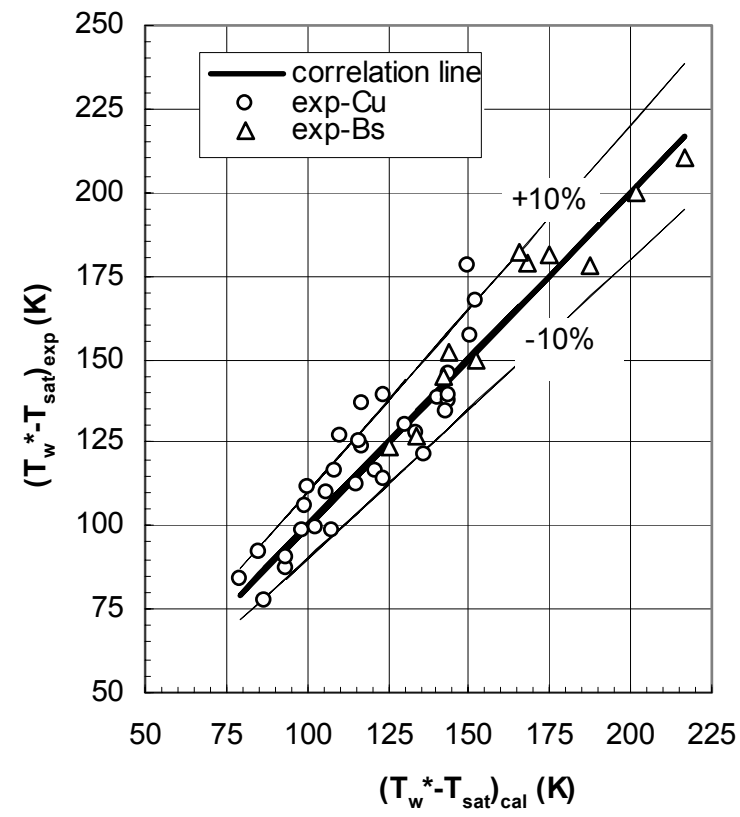

Fig. 8 Correlation for surface temperature $T_{\mathrm{w}}{ }^{*}$ at resident time $t^{*}$ (Rref. [6]) 


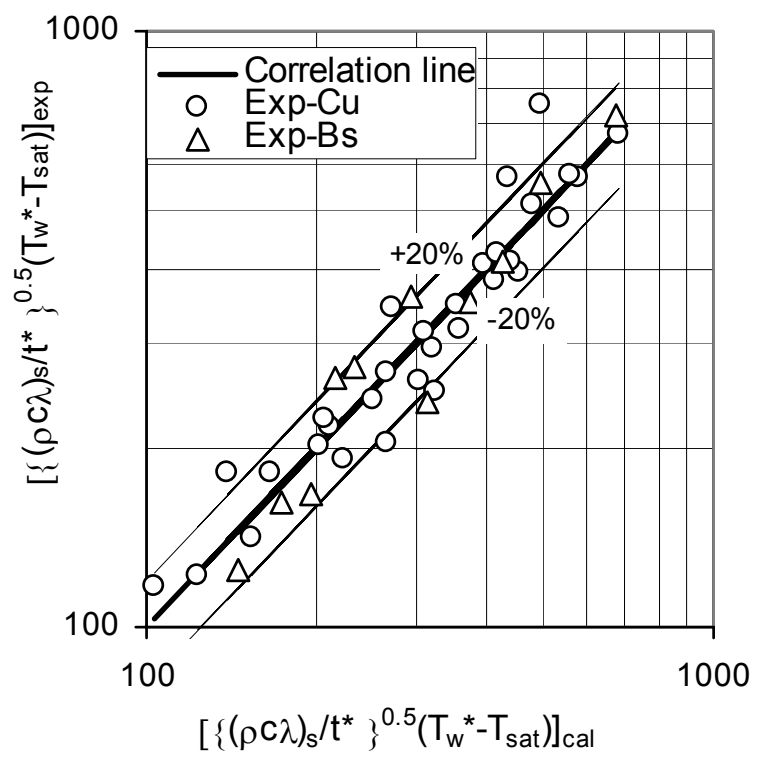

Fig. 9 Correlation for resident time $t^{*}$ (Rref. [6])

Figures 8 and 9 show the temperature of $T_{\mathrm{w}} *$ plotted against $(\rho c u)_{1}\left(T_{\text {sat }}-T_{\text {liq }}\right)$ based on Eqs.(2) and (3). The relationship between the resident time of $t^{*}$ and the surface temperature of $T_{\mathrm{w}}{ }^{*}$ surely exists as shown in Figs. 8 and 9. However, non-dimensional correlation has been not derived for them, yet.

Apart from the quench with impinging jet, incidentally, for the case of quench with mist, the surface temperature and heat flux at the resident time are given by Fig.10. Figure 11 shows the measures surface temperature and interface temperature calculated from Eq.(1) plotted against $G \Delta T_{\mathrm{sub}} / \beta$. The temperatures of $T_{\text {cr }}$ and $T_{\mathrm{SHL}}$ in Fig.10 are the critical temperature and the upper limit of superheated liquid temperature.

Figure 10 shows that a sharp drop in the surface temperature and a sharp rise in surface heat flux coherently occur, since the surface is uniformly quenched in the case of mist cooling. The surface temperature of $T_{\mathrm{w}} *$ increases linearly with $G \Delta T_{\text {sub }} / \beta$ within a small value of $G \Delta T_{\text {sub }} / \beta$, while beyond a certain value of $G \Delta T_{\text {sub }} / \beta$, the surface temperature is kept constant one close to the temperature of $\mathrm{T}_{\mathrm{SHL}}$.

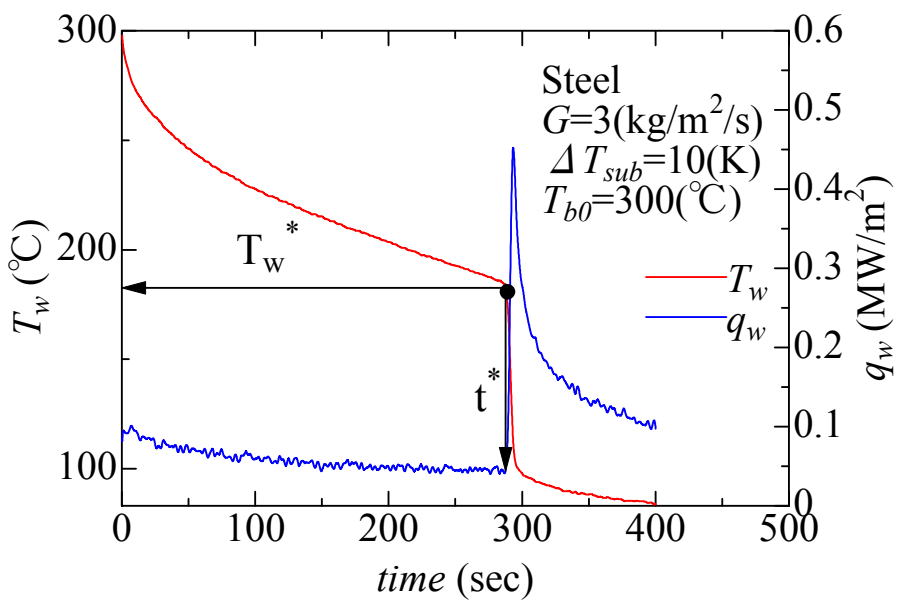

Fig.10 Surface temperature and heat flux during spray cooling of ethanol for carbon steel (mass flux $G=3 \mathrm{~kg} / \mathrm{m}^{2} / \mathrm{s}, \Delta T_{\text {sub }}=10 \mathrm{~K}, T_{b}=300{ }^{\circ} \mathrm{C}$ ) 


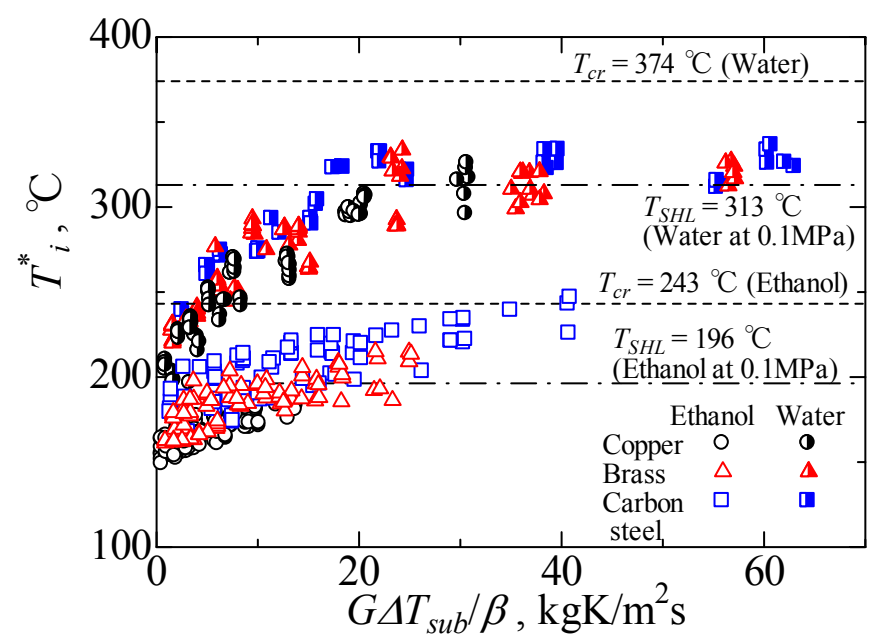

Fig.11 Surface temperature at resident time against $G \Delta T_{\text {sub }} / \beta \quad\left(\beta=\sqrt{(\rho c \lambda)_{s} /(\rho c \lambda)_{l}}\right)$

On comparison of Figs. 7 and 11, the interface temperature for $t^{*}<30 \mathrm{sec}$ shows significantly different trend between mist cooling and jet impingement cooling, that is unclear, yet.

\subsection{Heat transfer mode during movement of wetting front}

Figure 3 shows, as an example, the change in the surface temperature and heat flux during the movement of wetting front on the high temperature surface and the flow aspect near the wetting front after the establishment of stable wetting condition. It is found from Fig.3 that the maximum heat flux point appears slightly behind the wetting front. Filipovic et al. [11] called this as precursor phenomenon. The surface temperature sharply drops and inversely heat flux is rapidly increased between the wetting front and the maximum heat flux position. That is, the wetting condition shifts from unstable to stable one in the region between the wetting front and the maximum heat flux position. In other words, a transition boiling occurs in this region.

It is found from Fig. 2 that the maximum heat flux gradually decreases as the wetting front moves outward. The maximum heat flux is tentatively analyzed on the basis of equation (4) for critical heat flux with an impinging jet [13] and finally is correlated as shown in Fig. 11.

$$
\frac{q_{\max }}{q_{c}}=1-C \sqrt{(\rho c \lambda)_{l}} / \sqrt{(\rho c \lambda)_{s}}
$$

where

$$
\begin{aligned}
& \frac{q_{c}}{q_{c o}}=\frac{1+\sqrt{1+4 C_{1} J a}}{2} \quad, \quad J a=\frac{\rho_{l} c_{p} \Delta T_{s u b}}{\rho_{g} h_{\mathrm{lg}}} \\
& \frac{q_{c o}}{\rho_{g} h_{\mathrm{lg}} u}=0.221\left(\frac{\rho_{l}}{\rho_{g}}\right)^{0.645}\left(\frac{2 \sigma}{\rho_{l} u^{2}(D-d)}\right)^{0.343}(1+D / d)^{-0.364}
\end{aligned}
$$

where $q_{\mathrm{c}}$ is subcooed critical heat flux with impinging jet cooling, $d$ is diameter of heated surface and constant, $C_{1}$ is a function of liquid velocity and diameter of heated surface. The distance from the position of maximum heat flux to the center may correspond to the half of 
heated surface diameter.

It is worth mentioning that the critical heat flux predicted by Eq. (4) is reached under the condition that the critical heat flux occurs when heat is supplied from the infinite heat resource and the critical heat flux condition is not affected by any solid condition, namely is independent of solid side. The maximum heat flux appearing during the movement of the wetting front is well correlation by Eq. (4) with $C=5.5$ based on the correlation for the critical heat flux obtained by the steady heating as shown in Fig.12. This result may tell us that the maximum heat flux during the movement of wetting front is determined by a heat balance between capacities of heat supply form solid side and of cooling from liquid side and movement of the maximum heat flux is also induced by unbalance between both capacities. In addition the surface temperature is automatically fixed to balance both capacities, namely becomes equilibrium one.

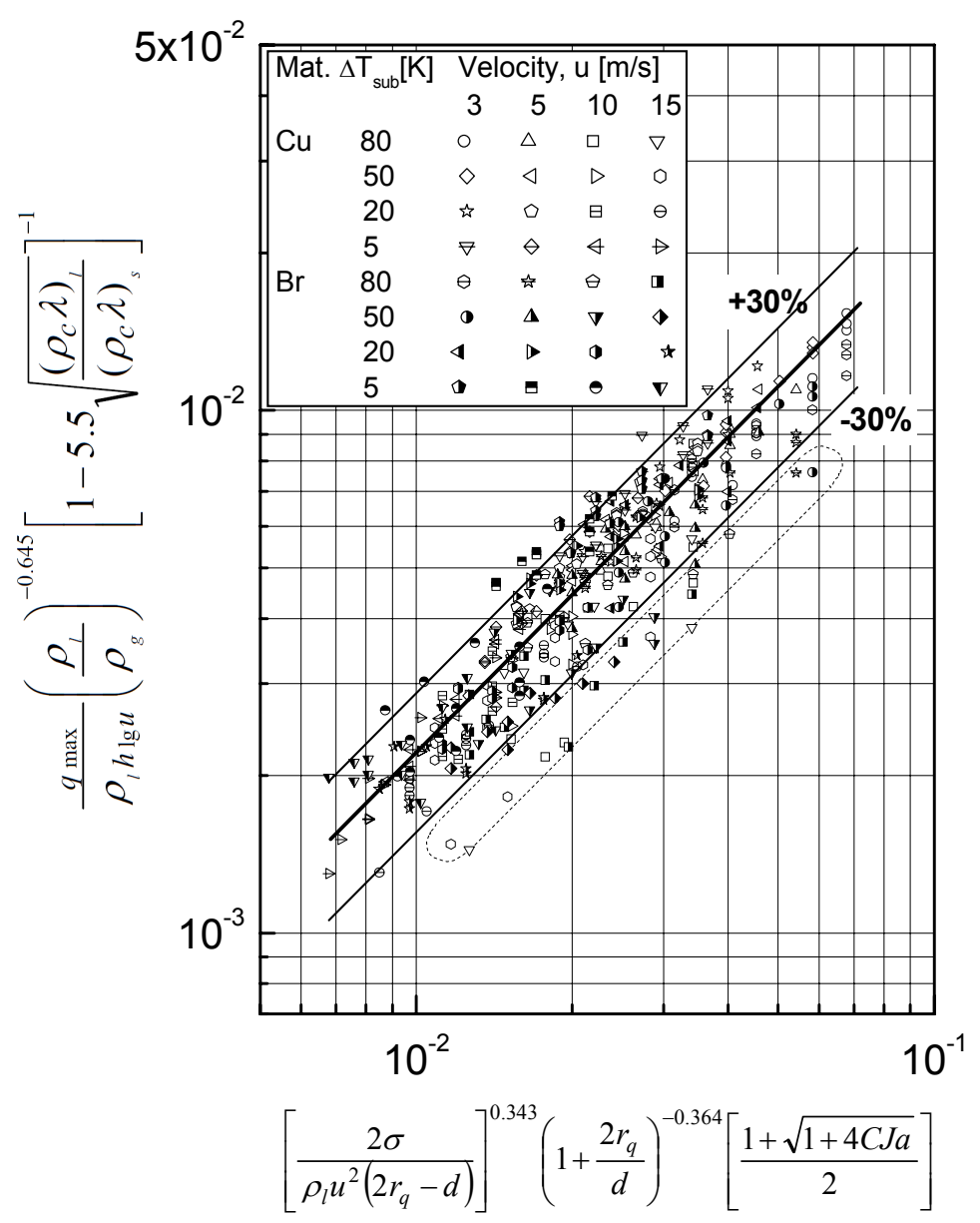

Fig.12 Estimation of maximum heat flux during movement of wetting front based on critical heat flux for steady state condition (Ref. [8])

The data encircled by broken line which is smaller than the predicted value, is obtained under the condition that the stable wetting appears immediately after jet strikes the hot surface. Accuracy of the measurement may lose compared with the other data which is measured after no movement of the wetting front is lasting for time more than a couple of minutes. In addition, the maximum heat flux data for carbon steel is predicted at less accuracy than that for copper and brass, since the wetting front quickly starts immediately after the jet impingement.

Incidentally, Hall et al. [14] proposed a correlation to predict the maximum heat flux 
during the movement of wetting front, when carbon steel at a temperature of $650{ }^{\circ} \mathrm{C}$ is quenched by impinging jet at a temperature of $25^{\circ} \mathrm{C}$.

$$
q_{\max }=q_{c o}\left(1+0.952\left(\rho_{l} / \rho_{g}\right)^{-0.888} \mathrm{Ja}\right)
$$

His quench has been done at quasi-state and gradually. Therefore, the maximum heat flux is thought to be almost reached close to a steady state.

Author recommends Eq. (4) being more reasonable than Eq. (5), since the maximum heat flux appearing during quench has been strongly affected by the capability of heat supply from solid side.

\section{Surface temperature for stable contact of liquid with high temperature surface}

The water on the surface is violently splashed away at a moment after water jet impinges on high temperature surface, as shown in Fig.1. During splashing of water, a high pitch sound is recorded that means vapor is generated on the surface. In addition, the frequency of the generated sound means that the wet and dry of the surface are alternatively repeated at a very short period. During this repetition, the average surface temperature gradually decreases with time and finally reaches a certain temperature, at which a stable contact between liquid and solid is established. Nucleation of the vapor which is formed by the direct contact between liquid and solid may be important phenomena to understand how the contact situation changes on the surface with time.

\subsection{Homogeneous nucleation}

According to homogeneous nucleation theory [15-17], there is a minimum required work for a certain size of cluster to change from liquid to vapor phases in the field of uniform liquid temperature. The state of the homogeneous nucleation can be determined by the minimum required work. However, the rate of nucleation extremely changes with a size of critical diameter of cluster. Table 1, for example, shows the rate of nucleation against the size of cluster for the case of water at a temperature of $20^{\circ} \mathrm{C}$ and one atmosphere. It is reported in Ref.[15] that a number of molecular in the cluster of the critical radius varies from 100 to 1000 and the temperature becomes $T_{\mathrm{L}} / T_{\mathrm{cr}}=0$. 885. Even though the critical radius hardly varies as shown in Table 1, the rate of nucleation changes over several digits. Under such situation, the rate of nucleation is arbitrarily chosen and the temperature for homogeneous nucleation is estimated for any rate of nucleation.

Table 1 Critical radius and homogeneous nucleation rate for water at $20^{\circ} \mathrm{C}$

\begin{tabular}{|c|c|c|c|c|}
\hline$r_{\mathrm{e}} \mathrm{nm}$ & 2.91 & 2.42 & 2.08 & 1.82 \\
\hline$J \mathrm{~m}^{-3} \mathrm{~s}^{-1}$ & $7.31 \times 10^{5}$ & $2.74 \times 10^{16}$ & $6.50 \times 10^{22}$ & $8.92 \times 10^{26}$ \\
\hline
\end{tabular}

\subsection{Heterogeneous nucleation}

Iida et al. $[18,19]$ reported that when the heated surface in pool liquid is rapidly heated at a rate of $k=10^{7}-10^{8} \mathrm{~K} / \mathrm{s}$, a tiny bubble like a kyabia is generated on the surface due to a homogeneous nucleation. The temperature distribution in the liquid is formed during rapid heating as given by the following equation.

$$
T(x, t)-T_{l}=4 k t i^{2} \operatorname{erfc}(x / 2 \sqrt{a t})
$$

On the other hand, the surface temperature and flow aspect change as shown in Fig.13 after the moment when the liquid contacts with high temperature surface. The temperature in Fig. 12 corresponds to the surface temperature estimated from Eq.(1) before beginning of solid-liquid contact on assumption that the interface temperature reaches the upper limit, 
$T_{\max }$ of superheated temperature for liquid. The flow aspect for the carbon steel in Fig.13 is obtained when the surface temperature for the carbon steel just crosses the temperature of $T_{\max }$. For the case of brass, the surface temperature is still higher than the temperature of $T_{\max }$ at the time when the surface temperature for the carbon steel just crosses the temperature of $T_{\max }$. Therefore, the flow aspect for brass still shows a violent and irregular splashed droplet like a chaos.

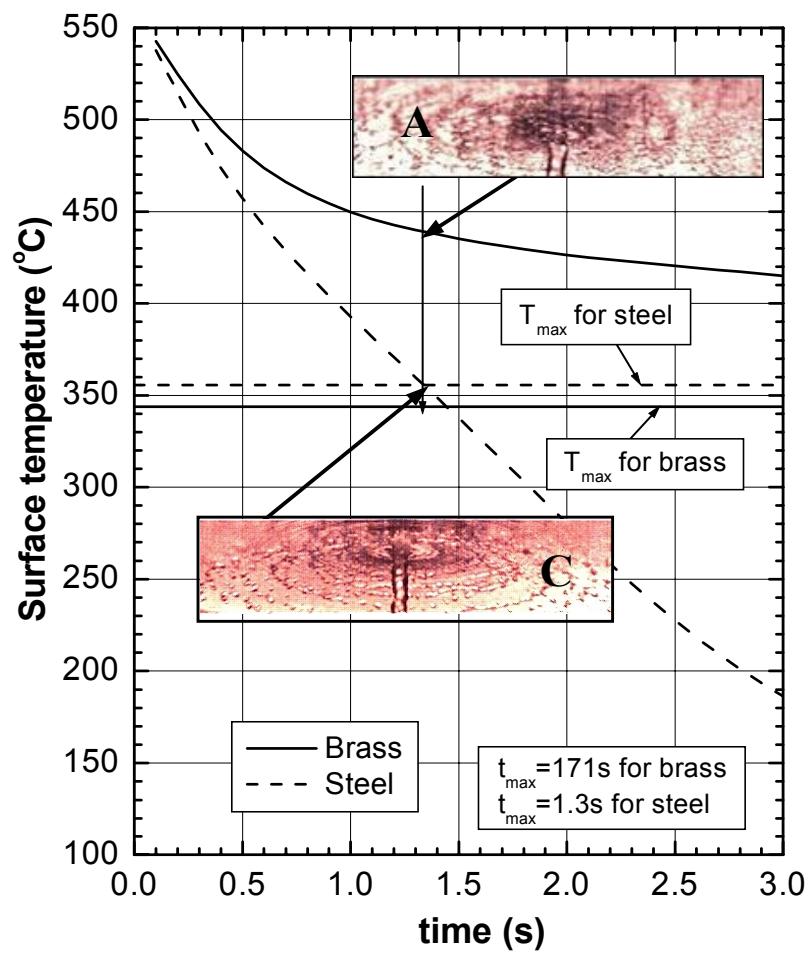

Fig. 13 Comparison of cooling curves for quenching $50^{\circ} \mathrm{C}$-brass and -steel blocks by $20 \mathrm{~K}-5 \mathrm{~m} / \mathrm{s}$ jets (Ref. [10])

The temperature distribution in the solid can be given using interface temperature at a moment of liquid-solid contact as:

$$
\frac{T(x, t)-T_{l}}{T^{*}{ }_{i}-T_{l}}=\operatorname{erfc}(x / 2 \sqrt{a t})
$$

If the size of the vapor cluster could be provided, then the average temperature for the cluster can be calculated using Eq.(7) as follows:

$$
T_{\text {lavg }}(x, t)=\frac{1}{x_{e}} \int_{0}^{x_{e}} T(x, t) d x
$$

where the distance $x_{\mathrm{e}}\left(x_{\mathrm{e}}=2 r_{\mathrm{e}}\right)$ is assumed to be equal to twice of the cluster diameter, $\mathrm{r}_{\mathrm{e}}$. The diameter of vapor cluster is, for example, given in Ref.[16] as follows:

$$
r_{e}=\frac{2 \sigma}{P_{\text {sat }}\left(T_{\text {lavg }}\right) \exp \left[v_{l}\left\{P_{\propto}-P_{\text {sat }}\left(T_{\text {lavg }}\right)\right\} / R T_{\text {lavg }}\right]-P_{\propto}}
$$

Figures 14 and 15 show how the temperature distribution in liquid, average temperature and the size of cluster change with time and depth in liquid when the carbon steel at the temperature of $350{ }^{\circ} \mathrm{C}$ is quenched by the liquid. It is found from Figs. 14 and 15 that the size of the cluster reaches the value of about $3.5 \mathrm{~nm}$ at the time of around $0.02 \mu \mathrm{s}$ at which 
the average temperature just reaches the homogeneous nucleation temperature. Under this condition, it might be possible to consider that violent splashed droplets are brought out by the formation of bubble nucleus. It is worth mentioning that it takes several $\mu$ s for the average temperature in the cluster to reach the homogeneous nucleation temperature when the liquid is heated by rapid increase in the surface temperature and that the increase in the average temperature for the cluster is relatively slower than that during quenching high temperature surface.

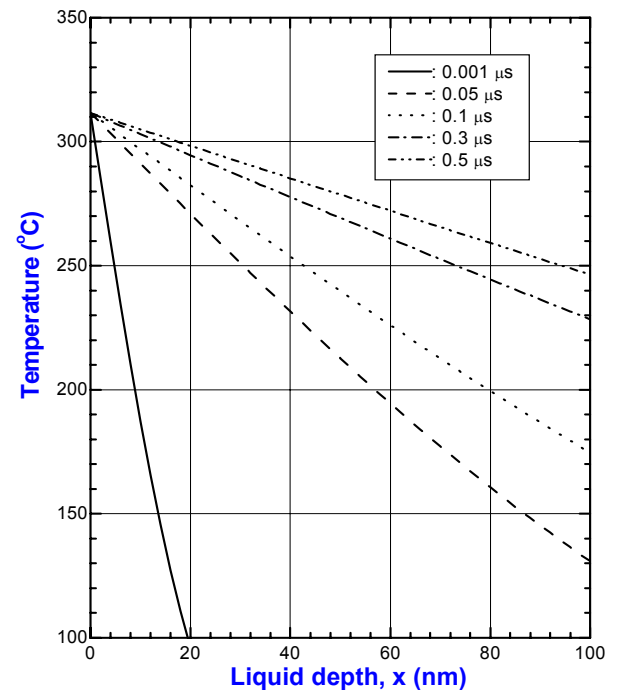

Fig.14 Temperature distribution of liquid at different time of contact for quenching $350^{\circ} \mathrm{C}$-Steel block by $80 \mathrm{~K}$ jet (Ref. [10])

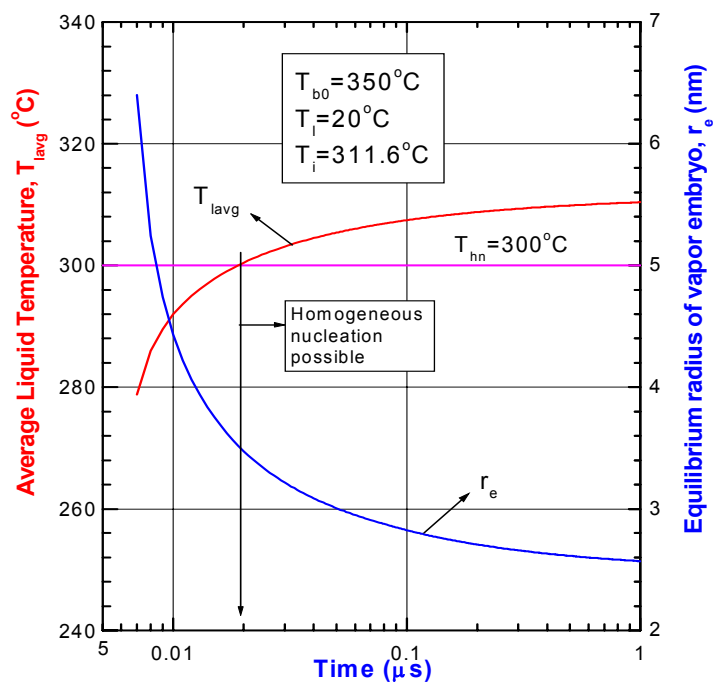

Fig.15 Variation of average liquid temperature and equilibrium bubble radius with contact time (Ref. [10])

\section{Characteristic regions in cooling curve}

The characteristics during the quench of high temperature solid with the impinging jet cooling can be categorized into three regions as shown in Fig. 16. That is, Region I, where no stable wetted area on the surface is established, Region II, where stable wetted area partially exists but does not enlarge itself, and Region III, where the wetted area starts moving forward higher temperature surface. On the other hand, the high 
temperature surface is uniformly quenched with a mist cooling, resulting into hardly appearance of Region II. As the result, Region I may quickly shift into Region III and the wetting temperature may appear around the superheated liquid temperature of $T_{\mathrm{SHL}}$.

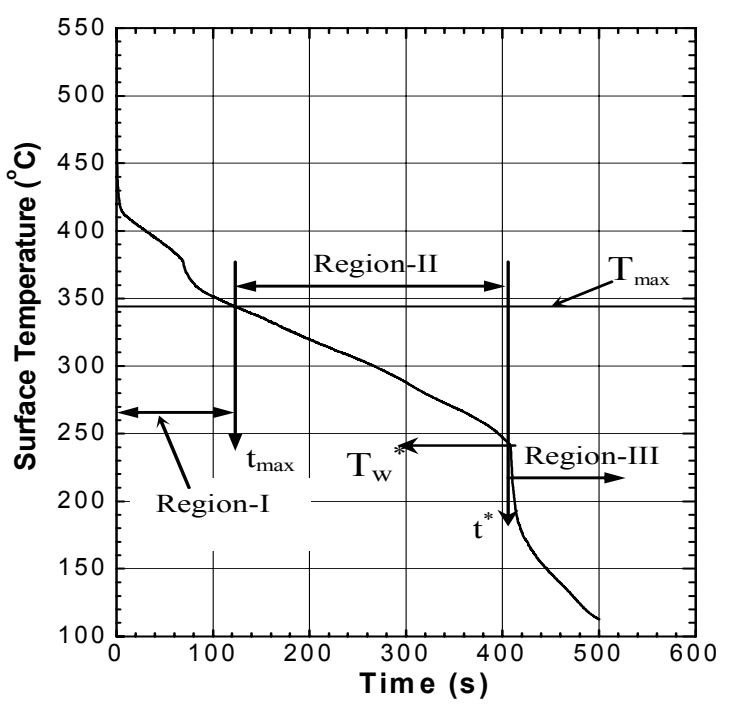

Fig.16 Characteristics regions during jet impingement quenching (Ref. [10])

\section{Summary}

There are many researches on quench of high temperature material in several cooling systems and on issues related to its wetting. In this review, the quench with impinging jet cooling system is focused and its heat transfer characteristics are discussed. The condition that liquid stably contacts with high temperature solid needs more study.

\section{Acknowledgement}

Author would like to appreciate Assoc. Professor Y. Mitsutake and Dr. P. Wood field of Saga University and Prof. A. Islam of Bangladesh University of Engineering and Technology for their helpful contributions to this research.

\section{References}

(1) M. Monde, H. Arima, W. Liu, Y. Mitutake and J. A. Hammad, An analytical solution for two-dimensional inverse heat conduction problems using Laplace transform, Int. J. of Heat and Mass Transfer, Vol.46 (2003) pp.2135-2148.

(2) J. Hammad, M. Monde, Y. Mitsutake and H. Arima, Determination of surface temperature and heat flux using inverse solution for two dimensional heat conduction, Thermal Science \& Engineering, Vol.10, No.2, (2002) pp.17-26.

(3) P. L. Woodfield, M. Monde and Y. Mitsutake, Implementation of an analytical two-dimensional inverse heat conduction technique to practical problems, Int. J. of Heat and Mass Transfer, Vol.49, (2006) pp.187-197.

(4) J. Hammad, Y. Mitsutake, and M. Monde, Movement of maximum heat flux and wetting front during quenching of hot cylindrical block, Int. J. of Thermal Sciences, Vol.43 (2004) pp.743-752.

(5) J. Hammad, M. Monde and Y. Mitsutake, Characteristics of heat transfer and wetting 
front during quenching by jet impingement, Thermal Science \& Engineering, Vol.12, No.1 (2004) pp.19-26.

(6) A. K. Mozumder, M. Monde and P. L. Woodfield, Delay of wetting propagation during jet impingement quenching for a high temperature surface, Int. J. of Heat and Mass Transfer, Vol.48 (2005) pp.5395-5407

(7) P. L. Woodfield, M. Monde and A. K. Mozumder, Observations of high temperature impinging-jet boiling phenomena, Int. J. of Heat and Mass Transfer, Vol. 48 (2005) pp.2032-2041.

(8) A. K.,Mozumder, M.,Monde, P. L.Woodfield, and A.,Islam, Maximum heat flux in relation to quenching of a high temperature surface with liquid jet impingement, Int. J. Heat Mass Transfer, Vol.49, (2006), pp.2877-2888.

(9) A.,Mozumder, P. L.,Woodfield, A. K.,Islam, and M.,Monde, Maximum heat flux propagation velocity during quenching by water jet impingement, Int. J. Heat Mass Transfer, Vol.50, (2007), pp.1559-1568.

(10) Md.A.,Islam, M.,Monde, P.L., Woodfield, and Y.,Mitsutake, Jet impingement quenching phenomena for hot surfaces well above the limiting temperature for solid-liquid contact, Int. J. of Heat and Mass Transfer, Vol.51, (2008), pp.1226-1237.

(11) J. Filipovic, F. P. Incropera, and R. Viskanta, Quenching phenomena associated with a water wall jet: 1 Transient hydrodynamic and thermal conditions, Experimental Heat Transfer, Vol.8 (1995) pp.97-117

(12)B. D. G. Piggott, E. P. White and R. B. Duffy, Wetting delay due to film and transition boiling on hot surfaces, Nuclear Engineering and Design, Vol.36 (1976) pp.169-181.

(13) M. Monde, K. Kitajima, T. Inoue and Y. Mitsutake, Critical heat flux in a forced convection subcooled boiling with an impinging jet, Heat Transfer-1994, Vol.7, (1994) pp.515-520.

(14)D. E. Hall, F. P. Incropera and R. Viskanta, Jet impingement boiling from a circular free-surface jet during quenching: part1- single-phase jet, ASME Journal of Heat Transfer, Vol.123 (2001) pp.901-910.

(15) C. F. Delale, J., Hruby and F. Marsik, Homogeneous bubble nucleation in liquids: The classical revisited, J. of Chemical Physics, Vol.118, (2003), 792-806.

(16) R. Cole, Boiling Nucleation, Advanced in heat transfer, 10 (1974), pp.86-116.

(17) Carey, V.P. (1992) Liquid-Vapor Phase-Change Phenomena, Taylor and Francis, New York, 1992 (Chapter 5).

(18) Y. Iida, K. Okuyama, and K. Sakurai, Peculiar bubble generation on a film heater submerged in ethyl alcohol and imposed a high heating rate over $10^{7} \mathrm{Ks}^{-1}$, Int. J. Heat Mass Transfer, Technical notes, Vol.36 (1993) pp.2699-2701.

(19) Y. Iida, K. Okuyama, and K. Sakurai, Boiling nucleation on a very small film heater subcooled to extremely rapid heating, Int. J. Heat Mass Transfer, Vol.37 (1994) pp.2771-2780. 